\title{
PLANIFICACIÓN FAMILIAR Y ABORTO EN PUERTO RICO: EXPLORANDO OPINIONES, CONOCIMIENTOS Y ACTITUDES
}

\author{
Giselle Rodríguez-Ocasio, Dimayra Rivera-López, \\ Carmen del Mar Morales Soto, Juan Pablo Benabe Vidal, \\ Milagros A. Méndez, Castillo ${ }^{I}$
}

\begin{abstract}
Resumen
La planificación familiar y la legalidad del aborto son temas que generan muchos debates dentro de la sociedad. Las posturas que asumimos sobre estos asuntos están relacionadas a nuestras creencias religiosas, ética, cultura, historia y al desarrollo e implementación de políticas públicas, entre otros. En la sociedad puertorriqueña se ha estudiado muy poco el tema de la planificación familiar y de la legalidad del aborto. El objetivo de esta investigación es explorar el conocimiento, las actitudes y las opiniones que tiene la sociedad puertorriqueña en torno a la planificación familiar y al uso de los fondos federales que se asignan a los grupos internacionales de planificación familiar que consideran el aborto como una opción (Política de Ciudad de México - PCM). Partiendo de un modelo mixto de investigación, se condujo una primera fase de carácter cualitativo con un alcance exploratorio y una segunda de carácter cuantitativo con un alcance correlacional. Obtuvimos una muestra por disponibilidad de 106 personas adultas que residen en Puerto Rico. De los hallazgos se desprende que ni los conocimientos ni las características socio-demográficas están relacionados a la actitud de las personas hacia la planificación familiar, a excepción de la ideología religiosa. Los hallazgos reflejan incongruencias entre el conocimiento sobre la planificación familiar y la derogación de la PCM, y la actitud hacia las mismas. A su vez, los resultados del estudio sustentan lo indicado en la literatura sobre el pobre conocimiento y el alto grado de confusión acerca de la legalidad del aborto en Puerto Rico.
\end{abstract}

Descriptores Planificación familiar, política de Ciudad de México, aborto, política pública

\begin{abstract}
Family planning and the legality of abortion are subjects that generate many debates within society. The positions we assume about these issues are related to our religious beliefs, ethics, culture, history and to the development and implementation of public policy. Puerto Rican society has not study family planning and abortion issues enough. The aim of this study is to explore the knowledge, attitudes and opinions of the Puerto Rican society towards family planning and the policy of United States that required all international non-governmental organizations refrain from performing or promoting abortion services as a method of family planning (Mexico City Policy - MCP). We carried out a mixed-method study with two phases: we conducted a first phase using a qualitative approach with an exploratory design; and a second phase using a quantitative
\end{abstract}

1 Departamento de Psicología, Universidad de Puerto Rico en Río Piedras 
approach with a correlational design. We conducted the study with a convenience sample of 106 adults who reside in Puerto Rico. The findings suggest that neither knowledge nor socio-demographic characteristics are related to the participant's attitude towards family planning, except religious ideology. The findings reflect inconsistencies between knowledge on family planning and the derogation of MCP, and the attitudes towards them. As well, as the literature suggest, the results of the study demonstrate poor knowledge and confusion about the legality of abortion in Puerto Rico.

Keywords: Family planning, Mexico City Policy, abortion, public policy

\section{Introducción}

Planificación familiar es un concepto que hemos escuchado desde la adolescencia en nuestras escuelas y por otros medios y que, en términos generales, se refiere a las prácticas que ayudan a evitar o favorecer el embarazo para, entre otros propósitos, adaptar el orden cronológico de los nacimientos a las edades de la madre y el padre, y determinar el número de embarazos deseados (Acosta et al., 1982). Entre esas prácticas se encuentran el uso de anticonceptivos, tratamientos de fertilidad, educación sexual y familiar, servicios de adopción, y otros (Acosta et al., 1982).

La planificación familiar es un concepto de interés tanto para las personas en edad fértil, como para los gobiernos y las agencias encargadas de crear políticas públicas. En el 2009, el presidente de los Estados Unidos, Barack Obama, firmó una orden ejecutiva para derogar la prohibición del uso de fondos federales a grupos internacionales que consideran el aborto ${ }^{2}$ como una opción de planificación familiar, restricción conocida como Política de Ciudad de México (PCM). Las posturas que asumen los gobiernos respecto al aborto son relevantes debido a que ha quedado demostrado que estos se realizan independientemente de su legitimidad y oportunidad de servicios, por lo que la política pública debe tomar en consideración la salud de las personas y en última instancia la sobreviviencia.

En Puerto Rico, el tema se hizo evidente cuando en el 2009, coincidiendo con la decisión de Obama, se disputó y luego se colgó el nombramiento de Joanne Vélez como Procuradora de la Mujer por reconocer el derecho al aborto. Esta controversia amplió el debate sobre la legalidad y posible apoyo económico del aborto. Estudios han demostrado que, a pesar de que el aborto es legal en Puerto Rico, es un tema confuso en el País, inclusive entre los y las profesionales de la salud (Azize y Henshaw, 2003; Mujeres Hoy, 2004). La ausencia de una visión salubrista, la desinformación, la influencia de discursos religiosos y los "códigos culturales vinculados al cuerpo" marcan de forma particular la relación que guarda la sociedad puertorriqueña con el tema del aborto (Azize y Avilés, 2009; Hernández-Angueira, 2008). Esto ocurre muy a pesar de que se ha demostrado que los programas de planificación familiar comprensivos aportan a disminuir los abortos en condiciones de riesgo y las defunciones maternas relacionadas. La derogación de la PCM permite, precisamente, promover diferentes opciones de planificación familiar que, además, ayudan a reducir el número de embarazos involuntarios y, por lo tanto, de abortos.

La mayoría de los países, incluyendo Puerto Rico, forman parte de un Programa de Acción sobre población, desarrollo y planificación familiar preparado en la Quinta Conferencia Internacional

2 En este artículo utilizamos la palabra aborto para hacer referencia, únicamente, al aborto inducido. 
sobre la Población y el Desarrollo (1994) de la Organización de las Naciones Unidas (ONU); Programa en el que los países deben estar trabajando activamente. Este estudio está dirigido a explorar algunos de los objetivos del Programa de la ONU: el acceso universal de salud reproductiva, que incluye planificación familiar y salud sexual; y la posibilidad de las mujeres a tener el control sobre su fertilidad.

El propósito principal de este trabajo fue proveer una experiencia de investigación a estudiantes sub-graduados del curso de Metodologías de Investigación en Psicología I y II (secciones 003 y 004) del 2009 de la Universidad de Puerto Rico, Recinto de Río Piedras. Además del propósito académico, esta investigación pretendía auscultar sobre diferentes aspectos o dimensiones de la planificación familiar y la derogación de la PCM. La mejor forma para que el estudiantado aprenda a realizar investigaciones éticas y significativas es teniendo una experiencia directa en el proceso. A su vez, entendemos que este estudio es relevante para la comunidad científica y para la sociedad por la vigencia del tema y por la importancia de desarrollar política pública en torno a la planificación familiar.

\section{Política pública internacional y Planificación Familiar}

Hace más de medio siglo que en la ONU se discuten temas relacionados a las poblaciones y su relación con el desarrollo económico de los países. Temas que, según pasan los años, se han ido vinculando a los derechos humanos, el cuerpo, lo público vis a vis lo privado, la relación estadoiglesia, entre otros. Hasta el momento, la ONU ha organizado seis cumbres o conferencias sobre este tema (Comisión Económica para América Latina y el Caribe [CEPAL], 2000).

A partir de la Primera Conferencia Internacional sobre Población y Desarrollo (CIPD-1) organizada por la ONU - Roma, 1954 - se promovió la creación de centros de capacitación en países en desarrollo para profesionales en temas de población y análisis demográfico. Una década después, se realizó en Belgrado - 1965 - la CIDP-2, contando con la presencia de profesionales en población se enfatizó en el análisis de la fecundidad como parte integral para crear una política para la planificación del desarrollo. En esta conferencia, comenzó a entenderse la llamada 'planificación familiar' como parte de una política para el control poblacional y de desarrollo de un país atada a las políticas demográficas, entendiendo estas como un "intento de modificar la estructura demográfica de una población por múltiples razones”, principalmente económicas (Torres, 2004, p. 105). En 1974 se celebró la CIPD-3 (Bucarest) siendo la primera de carácter intergubernamental, y en la que se impulsó el primer plan sustentado en las ideas desarrolladas en la conferencia anterior (1965) y que se conoció como el Plan de Acción Mundial sobre Población (PAMP) aceptado por los 137 países que participaron de la misma, entre ellos Estados Unidos (EUA). Esto dio pie, desde 1974, a la asignación de fondos federales a grupos internacionales con programas de planificación familiar.

En la década del ' 80 se celebró la CIPD-4 (1984) en la Cuidad de México, en la que se discutieron los hallazgos de las investigaciones realizadas sobre población en los últimos diez años y los datos provistos por los gobiernos para ampliar el PAMP. En temas de población se comenzó a discutir sobre derechos humanos individuales y familiares, salud y bienestar, empleo, educación, entre otros. A partir de dicha conferencia se creó la Declaración de la Política de Ciudad de México - PCM, 1984 -, documento que refleja la nueva política establecida por los Estados Unidos bajo la administración de Ronald Reagan. En esta política el aborto se vuelve un elemento 
inaceptable para la asignación de fondos a los programas de planificación familiar, incluyendo los fondos dirigidos a proveer información, orientación, educación e investigación. Cabe destacar que más de una década antes se vio en corte el caso Roe vs. Wade - 1973 - en el que se defendió el derecho a la intimidad y la legalidad del aborto durante el primer trimestre de gestación. Mientras que en la CIPD-3 - en el 1974 - se buscaban soluciones para tratar con el crecimiento poblacional y su relación con el progreso económico, en la CIDP-4 - 1984 - entraron en juego elementos de lo privado en temas de discusión de lo público. En estos elementos de lo privado vemos como empiezan a incidir razones morales en las decisiones que toma una nación sobre las políticas que establece.

La PCM perduró por 10 años aproximadamente en Estados Unidos, hasta que el Presidente Bill Clinton la derogó en el 1993. Un año después se celebró en el Cairo la CIPD-5 - 1994-, conferencia en la que 180 países aprobaron un nuevo Programa de Acción para guiar la gestión nacional e internacional en el área de población y desarrollo por los próximos 20 años (Véase $\$ 3$ de Introducción). Este plan hace evidente que las metas ya no son a nivel macro sino que van dirigidas a responder a las necesidades individuales según los planteamientos de los derechos humanos; sin embargo, no olvidemos que estos están en constante discusión y cuestionamiento por el devenir mismo de las personas y las sociedades (Daros, Contreras Nieto y Secchi, 2007).

Luego de la CIPD-5 - 1994 - se han celebrado varias conferencias internacionales organizadas por la $\mathrm{ONU}$ en las que se ha revisado la implementación del Programa de Acción. A pesar de que en la CIPD-5 las discusiones y propuestas no fueron evidentemente en torno a asuntos demográficos ni poblacionales, el discurso en las conferencias sub-siguientes ha tenido un claro matiz sobre estos temas (Torres, 2004). Evidencia de lo antes expuesto es el análisis realizado por Torres (2004, p. 158) quien, acertadamente, lo recoge en las palabras que pronunció en el 2009 el Secretario General de las Naciones Unidas, Kofi Annan:

Todos los Estados ahora comprenden que si pretenden proveer adecuadamente a la salud y a la educación (...) necesitan incorporar políticas de población en sus estrategias de desarrollo. (...) Desde El Cairo, el mundo entiende que debemos estabilizar la población de este planeta. (...) Cada ser humano aspira a la salud, a la seguridad y a la dignidad. Esta es la esencia de los derechos humanos. Y ahora nosotros comprendemos que los derechos reproductivos y sexuales son una parte esencial de estos derechos (citado por Torres, 2004).

En fin, se espera que para el 2015 se hayan cumplido los objetivos dirigidos a un acceso universal a la salud reproductiva, educación primaria para toda la población, reducir las tasas de mortalidad de infantes y la mortalidad materna y elevar la esperanza de vida al nacer a 75 años o más (CEPAL, 2004). Además, se espera adelantar en la equidad de género, eliminar la violencia contra las mujeres y asegurar la posibilidad de estas últimas a tener el control sobre su fertilidad (CEPAL, 2004).

\section{Política pública de Estados Unidos de América}

Estados Unidos de América ha condicionado la ayuda a los programas de planificación familiar a su uso, con énfasis en el tema del aborto. Como hemos mencionado, la Administración Reagan, retiró los fondos a las instituciones que consideraban el aborto entre sus opciones de planificación familiar con la PCM - 1984. De acuerdo a Wallace (2008), esta Política fue parte de una serie de 
cambios encaminados a reformar el Estado de Beneficencia (Welfare State) vigente al momento. La definición de Estado de Beneficencia sugiere que el gobierno debe tomar un papel clave en la protección y desarrollo económico-social de sus ciudadanos basándose en la igualdad de oportunidades, la distribución equitativa de las riquezas y la responsabilidad pública de ayudar a otros con pocos recursos para hacerlo por sí mismos (Enciclopedia Británica, 2011). Reagan planteaba que las ayudas económicas a las clases de escasos recursos debían ser reducidas, sin aumentar el costo de vida de dicha clase (On The Issues, 2010). En su campaña fomentó la idea de que muchas de las personas pobres lo son por sus propias decisiones, justificando así las reformas con las que cortaría los fondos públicos dirigidos a ese sector. Según sus planteamientos, para mejorar la economía del País, estos fondos serían redistribuidos a las grandes corporaciones y bancos que apoyarían económicamente al gobierno. Estas decisiones fueron apoyadas por la recesión del '82 y que Reagan achacaba al Estado de Beneficencia (Smitha, 2011).

El 22 de enero de 1993, día del vigésimo aniversario del caso Roe vs. Wade, Bill Clinton derogó la PCM en un esfuerzo por ayudar a los sectores pobres a integrarse al mundo laboral entendiendo que dicha política socavaba sus esfuerzos de promover prácticas de planificación familiar eficaces y seguras (Clinton, 1993). Esto, partiendo de la idea de la planificación familiar como una forma de asegurar que las personas pudieran integrarse al mundo laboral y no dependieran del Estado de Beneficencia. George W. Bush, siguiendo la plataforma de su partido, restituyó la PCM en el 2001, también un 22 de enero. En el 2009, Barack Obama derogó nuevamente la PCM, no un 22 de enero sino al día siguiente; esto podría reflejar un mensaje implícito de despolitizar el tema y asumir posturas menos ideológicas entorno al mismo. En fin, todo parece indicar que los presidentes republicanos han apoyado la PCM y los demócratas la han derogado. Estos cambios de política reseñan el papel protagónico que ha asumido EUA en torno a la planificación familiar (Torres, 2004).

En síntesis, un tema controversial es la asignación de fondos federales a grupos internacionales que consideran el aborto como una opción de planificación familiar. El tema ha ido evolucionando de una discusión internacional cuyos objetivos giraban en torno a asuntos demográficos a una discusión que cambia su foco hacia las familias y las personas, aunque para lograr los objetivos iniciales de control poblacional y desarrollo económico. En este asunto es evidente el impacto y el poder del Estado en las decisiones individuales, el control sobre el cuerpo, los derechos humanos, entre otros. Asunto que, además de estar altamente ideologizado y politizado, tiene fuerte impacto en Puerto Rico por nuestra relación con EUA.

\section{Política pública en Puerto Rico}

En Puerto Rico la legalidad del aborto es confusa. Por un lado, el estado de derecho reconoce el aborto como una práctica legal bajo la jurisprudencia federal. Por el otro, el Código Penal de Puerto Rico, Ley Núm. 149 del 18 de junio de 2004 (Secc. 3, art. 111 al y 114), prohíbe el aborto, ya sea voluntario o forzado, con excepción de salvar la salud o vida de la madre; aunque esto queda sin efecto porque Puerto Rico se rige por la ley federal. El Portal de las mujeres latinoamericanas (Mujeres Hoy, 2004), realizó una encuesta sobre este tema y encontró que existe:

...un doble discurso respecto a la interrupción voluntaria del embarazo deja a las mujeres puertorriqueñas en un espacio de decisión ambiguo y estigmatizado. Mientras el estado de derecho reconoce al aborto como una práctica legal, un 30 por ciento de ellas sigue creyendo que es un acto ilícito. (I1) 
Esto se debe al abismo existente entre la jurisprudencia vigente en Puerto Rico y la política gubernamental. Azize, directora de Salud promujer y especialista en temas de género, expresó en Mujeres Hoy (2004) que, "las opiniones personales de los gobernantes y la presión ejercida por grupos religiosos ha imperado sobre las decisiones de los tribunales en los Estados Unidos y Puerto Rico". (\$6)

Aunque el aborto se legalizó en Puerto Rico por su condición colonial, las protestas de líderes políticos, religiosos y culturales fueron evidentes y con fuerza suficiente para revocar la aplicación a Puerto Rico (Azize y Avilés, 2009). Aún así, en Puerto Rico se dieron dos casos judiciales en los que el Tribunal Supremo dictaminó que la medida aprobada en el caso Roe vs. Wade tiene vigencia en Puerto Rico, confirmando así la legalidad del aborto: el caso [Acevedo] Montalvo v. [Hernández] Colón en 1974, y el caso Pueblo v. Duarte Mendoza en 1980 (Colón-Warren, 2001). Paradójicamente, la posición que tomó el Tribunal Supremo de Puerto Rico fue más liberal que el de Estados Unidos al no limitar la legalidad del aborto al primer trimestre (Azize y Avilés, 2009). Esto crea una situación contradictoria en la que Puerto Rico, simultáneamente, tiene una de las leyes sobre aborto más liberales y una de las políticas públicas más conservadoras. De hecho, la incidencia de abortos legales en Puerto Rico es más baja que en otros países con políticas más restrictivas e inclusive que en países en los que es ilegal, diferencia que se exacerba en las adolescentes (Azize y Avilés, 2009). De acuerdo a Azize y Henshaw (2003), mientras que en Puerto Rico se realizaron 18 abortos por cada 1,000 mujeres en edad reproductiva (2001), en Estados Unidos se realizaron 21 por cada 1000 (2000). En Guyana, por cada aborto realizado en Puerto Rico se realizaron cuatro. Inclusive en República Dominicana, aún cuando el aborto es ilegal, se estima que se realizan 47 abortos ilegales por cada 1,000 mujeres (1990). Esto puede deberse a: la influencia de la Iglesia, las desigualdades, confusión, inaccesibilidad (costos, facilidades, procedimientos), entre otros.

Un hecho actual que corrobora la posición ambigua de la sociedad puertorriqueña y que hizo que el tema cobrara vigencia fue la controversia en torno al nombramiento de Joanne Vélez como Procuradora de la Mujer (2009) tras reconocer el derecho al aborto que le dio el Tribunal Supremo de Estados Unidos a la mujer y que es reconocido en Puerto Rico. Finalmente, el nombramiento fue colgado el 30 de abril de 2009 (NotiUno, 2009a) ocasionando que diferentes grupos denunciaran que los criterios utilizados por el Senado no siguieron lo estipulado por la Ley de la Oficina de la Procuradora de las Mujeres, Ley Núm. 20 del 11 de abril de 2001, para el puesto de Procuradora. "En una crasa violación al principio constitucional de separación entre Iglesia y Estado, las opiniones religiosas de las y los senadores se utilizaron para rechazar este nombramiento" (Movimiento Amplio de Mujeres de Puerto Rico [MAMPR], 2009, J4).

Cuatro meses después que colgaron el nombramiento de Joanne Vélez, el Senado confirmó a Yvonne Feliciano ignorando las críticas de varios de sus componentes y de grupos como el Caucus de la Mujer del Movimiento Socialista de Trabajadores, del MAMPR, entre otros (NotiUno, 2009b). Una de las razones expuestas para que estos grupos rechazaran a Yvonne Feliciano como Procuradora fue que ella "nunca estableció posición alguna sobre temas importantes como el libre uso de anticonceptivos, el aborto, las uniones de hecho" (NotiUno, 2009b, I2), entre otros temas. Estos grupos entienden que para poder defender los derechos de las mujeres puertorriqueñas y ser capaz de impulsar políticas públicas que las tomen en consideración es necesario conocer los problemas que las aquejan y asumir una posición ante ellos (MAMPR, 2009). Todavía al día de hoy (2010) mujeres como las de La Juntilla (Indymediapr.org, 2010), punto focal en Puerto Rico 
de la Campaña por una Convención Interamericana por los Derechos Sexuales y Reproductivos, le reclaman al gobierno que "cumpla con su responsabilidad de educar a la población femenina en relación a su salud sexual y reproductiva" (I1). Además, denuncian que el estado "se ha convertido en un fotuto de las organizaciones religiosas fundamentalistas, dejando atrás su deber de educar a nuestras niñas y mujeres. [...] Aquí, donde el aborto es legal, el Estado tiene la responsabilidad de educar para prevenir” (I/2 y 3). Pero como destacó Zuly García, portavoz de La Juntilla, en Puerto Rico el "gobierno no sólo dificulta el trabajo de nuestras organizaciones, sino que se hace invisible a la hora de desarrollar programas de prevención y divulgación de información” (Indymediapr.org, 2010, \$5).

De la exposición anterior se desprende que los argumentos en contra del aborto y, por tanto, a favor de la PCM están sustentados en ideales moralistas y religiosos, y no por razones salubristas. A esta situación se le suman diferentes obstáculos para obtener servicios de aborto, entre ellos: desinformación, escasez de proveedores, distribución desigual de servicios y dificultades económicas. Es interesante observar cómo, en Puerto Rico, aun cuando el aborto es legal, la sociedad no lo ha asumido así por razones sociales, culturales e históricas. Las pautas culturales y sociales sobre la sexualidad en Puerto Rico están estrechamente vinculadas a su historia colonial, primero en relación a España y luego en relación a EUA (Hernández-Angueira, 2008). Respecto a la cultura hispano-católica, vigente hoy, Hernández-Angueira (2008) señala que "reprodujo en Puerto Rico los valores, las creencias, las prácticas y todo un discurso colonial con respecto a la sexualidad y al género, que (...) se incrusta profundamente en la psiquis y la conducta del colonizado" (p. 131). Ver que en las páginas amarillas de la guía telefónica no se permite el uso de la palabra aborto y que se exige a las clínicas de aborto usar eufemismos tales como 'soluciones para embarazos problemáticos’ es muestra de ello (Azize y Avilés, 2009). Es preocupante que las estimaciones a nivel internacional de defunciones maternas resultantes del aborto en condiciones de riesgo ascienden a 68,000 (Organización Mundial de la Salud, 2004). Esta situación podría prevenirse con la ampliación y el mejoramiento de los programas de planificación familiar para que fomenten el uso de métodos anticonceptivos eficaces y reduzcan el número de embarazos involuntarios y abortos. Si se previenen los embarazos involuntarios, se podrían ahorrar recursos de los servicios de salubridad que se necesitarían para tratar las complicaciones de los abortos peligrosos, ocasionados en parte por la desinformación (Population Reports, 1999). Azize y Avilés (2009) destacan que en Puerto Rico se han realizado muy pocas investigaciones que exploren la relación de factores políticos, económicos y culturales que influyen en las prácticas reproductivas.

\section{Los objetivos de esta investigación recogen algunos de estos aspectos:}

1. Auscultar el conocimiento que tienen las personas adultas que residen en Puerto Rico sobre planificación familiar (PF) y Política de Ciudad de México (PCM).

2. Describir las actitudes y opiniones de los y las participantes sobre PF y la PCM.

3. Conocer las bases y supuestos de dichas opiniones y actitudes.

4. Explorar la relación entre actitud hacia la PF y la PCM y el conocimiento de los y las participantes y sus características socio-demográficas.

5. Describir las alternativas que proponen los y las participantes a la PF. 


\section{Metodología}

Realizamos el estudio con una metodología mixta combinando los enfoques cuantitativos y cualitativos. Según una de las acepciones de la metodología mixta (Creswell y Plano-Clark, 2007), unimos los datos cualitativos que obtuvimos por medio de una entrevista semi-estructurada en la fase I del estudio con los datos cuantitativos que recogimos en la fase II por medio de una serie de cuestionarios. Con estas técnicas buscábamos auscultar las opiniones, actitudes y conocimiento de los y las participantes acerca de los temas centrales de esta investigación (Fase I), y explorar la relación entre estas variables y con algunas de sus características socio-demográficas (Fase II).

En la fase I analizamos los resultados de la entrevista mediante un análisis de contenido sobre conocimientos y opiniones utilizando el programa Atlas-ti. Luego, en la fase II, administramos unos cuestionarios para medir actitud, conocimiento y opiniones. En esta fase, analizamos los datos socio-demográficos y opiniones por medio de un análisis descriptivo de distribución de frecuencia y un análisis estadístico inferencial por medio de una prueba no paramétrica ji cuadrada para verificar las hipótesis utilizando el Programa SPSS versión 17. Aunque analizamos los datos de manera separada, realizamos una discusión integrada de los mismos, siguiendo las pautas de Creswell y Plano-Clark (2007).

\section{Participantes}

Los criterios de inclusión de la muestra para ambas fases fueron: ser mayor de 21 años y residir en Puerto Rico. Para los análisis de la fase I la muestra fue de 10 participantes de los cuales: cuatro eran mujeres y seis eran hombres. En términos de edades, seis se encontraban entre los 21 y 26 , uno tenía 34 y los restantes tres se encontraban entre los 44 y los 50. Seis eran de nacionalidad puertorriqueña, vivían en el área metropolitana y pertenecían a alguna religión. La muestra de la fase II se compuso de 96 participantes, para sus características demográficas vea la Tabla 1.

\section{Tabla 1}

\section{Características sociodemográficas de los y las participantes de la fase II}

\section{Características Socio-demográficas}

\begin{tabular}{lcccc}
\hline Sexo & Edad & $\begin{array}{c}\text { Afiliación } \\
\text { Religiosa }\end{array}$ & $\begin{array}{c}\text { Ideología Política } \\
\text { para EEUU }\end{array}$ & Nivel de Educación \\
\hline F (67\%) & $21-39(67 \%)$ & Catolicismo (52\%) & Republicano (10\%) & $\begin{array}{c}\text { Estudios (75\%) } \\
\text { Pos-secundarios }\end{array}$ \\
M (33\%) & $40-60(31 \%)$ & Protestante (26\%) & Demócrata (49\%) & $\begin{array}{c}\text { Escuela Superior (25\%) } \\
\text { o intermedia }\end{array}$ \\
M y F & $610+(2 \%)$ & Otra (3\%) & Ninguna (39\%) & Ninguna (19\%) \\
No opinaron & $2 \%$ & & & \\
\hline
\end{tabular}




\section{Instrumentos}

Para la fase I diseñamos una guía de preguntas para la entrevista y para la segunda fase dos instrumentos: el Inventario de actitudes hacia la PF y la PCM y el Cuestionario de conocimientos y opiniones sobre PF y la PCM. El primero consta de 20 reactivos a base de una escala Likert. El nivel de confiabilidad del instrumento alfa de Cronbach es de .598 .

El segundo instrumento se divide en dos partes: 1. Conocimientos sobre PF y PCM; y 2. Opiniones relacionadas a la PF y PCM. La primera parte se compone de 20 reactivos para contestar con una escala Likert y obtuvo una confiabilidad alfa de Cronbach de .890 . La segunda parte tiene seis reactivos donde se circulaba la respuesta que mejor contestara la pregunta o enumeraba según nivel de importancia.

\section{Procedimientos}

Una vez el estudio fue aprobado por el Comité Institucional para la Protección de Sujetos Humanos en la Investigación (CIPSHI) de la Universidad de Puerto Rico del Recinto de Río Piedras, nos reunimos con cada participante por aproximadamente una hora para discutir y completar la Hoja de Consentimiento Informado, la Hoja de Datos Socio-demográficos y realizar la entrevista semi-estructurada, la cual fue grabada en audio o video con el propósito de facilitar la trascripción.

Para la fase II, además de los documentos iniciales, le administramos el Inventario de Actitudes seguido por el Cuestionario Sobre Conocimientos y Opiniones. El espacio para recoger los datos fue seleccionado por los y las participantes. Al final de la colaboración con el estudio, les hicimos entrega de una hoja de agradecimiento con información sobre la PF y la PCM.

\section{Resultados}

\section{Fase I}

Realizamos un análisis de contenido de las entrevistas realizadas y desarrollamos unas categorías en las que agrupamos las expresiones de los y las participantes.

Categoría: Conocimientos. La mayoría indicó no conocer sobre el tema, algunos adjudicando el desconocimiento a la falta de acción del gobierno. Aún aquellas personas que dijeron conocer sobre el tema, especificaron que su conocimiento era limitado y lo vincularon directamente a controversias, en algunos casos políticas, sobre el aborto. Algunas de las expresiones fueron:

P: No tengo información clara y precisa ya que esto no es un tema que el gobierno divulga a plenitud y a puertas abiertas.

P: He escuchado algo pero no estoy cien por ciento al tanto, (...) creo Bush estaba en contra del aborto y por lo tanto estaba todo eso parado. 
Categoría: Actitud. La mayoría de los y las participantes asumieron una postura respecto a la PCM, a pesar de su poco conocimiento. Las personas que opinaron favorablemente lo vincularon a la prevención del aborto. Una de las personas compartió:

P: (...) entiendo que si es dirigido a un plan integrado (...) donde se justifique que se haya dado una orientación debida, yo entiendo que se pueden asignar (...), sino que t ambién se les está ofreciendo unas herramientas (...) para que entonces también eviten el aborto.

Sin embargo, la mayoría se expresó en contra del aborto y entendían que derogar la PCM era un voto a favor del mismo. Expresaron que el mismo no es una práctica de planificación y que por tanto no debe ser considerado como parte de la PF.

Categoría: Supuestos/bases de las opiniones. Agrupamos las expresiones sobre sus posturas en las siguientes sub-categorías: histórico-culturales, políticas, religiosas, legales y económicas. Algunas personas fueron explícitas al indicar que su opinión era desfavorable a la PF por razones religiosas y morales. Algunas expresiones fueron:

P: En mis principios religiosos, en que una persona con vida no se le debe coartar porque es un ser humano.

Categorías: Alternativas a la planificación familiar. De esta categoría surgieron tres sub-categorías: orientación, diferentes métodos anticonceptivos y alternativas relacionadas a los ingresos de las personas. Una de las expresiones fue:

P: Bueno, obviamente, protección, darle información sobre las enfermedades transmisible sexualmente, orientación, mucha orientación y mucha educación, no solamente en palabras, sino que sea vivencial, porque de orientaciones hay muchos papelitos por ahí y nadie les hace caso.

En general, las personas participantes favorecieron la utilización de los fondos para la creación de programas preventivos en los que se eduque a las personas sobre la PF. Solo una persona propuso la abstinencia como primera opción, mientras que otro propuso la adopción. Ninguno estuvo de acuerdo con el aborto como alternativa de PF.

\section{Fase II}

A partir de los hallazgos de las entrevistas realizadas en la primera fase, construimos un cuestionario sobre opiniones en torno a la PF y la PCM y dos escalas: una sobre conocimiento y una sobre actitud. Para que el análisis de los resultados de la variable actitud sea más manejable deseamos aclarar que partimos de la premisa de que una planificación familiar (PF) comprensiva y con una visión salubrista queda coartada por la PCM. Por tanto, tener una actitud favorable a la PF significa estar en contra de la PCM, mientras que una actitud desfavorable a la PF implica estar de acuerdo con dicha Política. Obtuvimos que el 54\% manifestó una actitud desfavorable, mientras que el $46 \%$ mostró una actitud favorable. 
De acuerdo a los análisis descriptivos para la variable conocimiento el $56 \%$ manifestó tener poco o ningún conocimiento y el $44 \%$ tener algún o mucho conocimiento. En cuanto a las opiniones, el 86\% indicó que la asignación de fondos federales para programas de planificación familiar es un tema relevante para Puerto Rico, y al preguntarles directamente, el 63\% de la muestra apoyó la decisión del Presidente Obama de derogar la PCM, a pesar de no conocerla bien.

A personas participantes se les pidió que enumeraran en orden de importancia que vertiente debería tener más peso al considerar medidas sobre PF. El 33\% seleccionó en primer lugar una visión salubrista. Sin embargo, estuvo seguida por la vertiente moral (17\%) y la vertiente económica con igual apoyo (17\%). El 56\% ubicó en último lugar la vertiente política. Entre las alternativas propuestas para la planificación familiar, el $45 \%$ seleccionó como método número uno la abstinencia, seguida por diferentes métodos anticonceptivos. La opción menos aceptada fue el aborto $(74 \%)$.

En términos de la política pública en Puerto Rico, el $60 \%$ se inclinó por la prevención. Referente a los fondos federales asignados a grupos internacionales de PF en temas vinculados al aborto, el $89 \%$ estuvo de acuerdo con que se utilizaran para orientación y educación, el 63\% para investigación, $38 \%$ con que utilicen para ofrecer información y el 36\% para apoyar clínicas legales de aborto.

Realizamos análisis correlaciónales de ji cuadrada para explorar la relación entre actitud y cada una de las variables edad, sexo, nivel de educación, ideología política para Estados Unidos y conocimiento, entre las que no hubo relaciones estadísticamente significativas. Sin embargo, encontramos una relación estadísticamente significativa, $\mathrm{X} 2(9, \mathrm{~N}=96)=28.81, \mathrm{p}=.001$, entre afiliación religiosa y actitud (ver tabla 2).

Tabla 2

Actitud hacia la PF (y la derogación de la PCM) según la afiliación religiosa

Afiliación Religiosa

\begin{tabular}{lccccc}
\hline $\begin{array}{l}\text { Actitud PF } \\
\text { Altamente }\end{array}$ & Católico & Protestante & Otra & Ninguna & Total \\
desfavorable & 9 & 2 & 1 & 2 & $14(14.6 \%)$ \\
Desfavorable & 23 & 13 & 0 & 2 & $38(39.6 \%)$ \\
Favorable & 15 & 5 & 2 & 4 & $26(27.1 \%)$ \\
Altamente favorable & 3 & 5 & 0 & 10 & $18(18.7 \%)$ \\
\hline Total & $50(52 \%)$ & $25(26 \%)$ & $3(4 \%)$ & $18(19 \%)$ & $96(100 \%)$
\end{tabular}

\section{Discusión}

Entre los hallazgos más considerables que reflejó el estudio es que el conocimiento sobre la planificación familiar (PF), el aborto y la PCM son muy limitados, lo que es congruente con las conclusiones de otros estudios (Azize y Avilés, 2009; Hernández-Angueira, 2008; Villarini-Baquero, 1994). En aquellas personas que dijeron conocer sobre el tema, se evidencia incongruencias entre 
lo que conocen y la actitud que asumen ante el mismo. La pobre relación entre conocimiento y actitud observada en esta investigación es consistente con otras investigaciones psicológicas - realizadas con muestras diferentes a través del tiempo $(1987,1994$ y 2009) - en las que se ha visto que tener conocimiento sobre alguna práctica en particular no necesariamente influye en la actitud de las personas (Chéveres-Rodríguez, 1987; Villarini-Baquero, 1994).

El poco conocimiento sobre PF y la PCM, puede deberse al hecho de que éste está vinculado a otros factores como: el interés, la necesidad, las obligaciones, el posicionamiento, entre otros. Los resultados de nuestro estudio parecen ser reflejo del desconocimiento y la confusión general en Puerto Rico acerca de la legalidad del aborto que va de la mano de lo que Gutiérrez (2004) llamó un "profundo silencio social". La controversia y el silencio ante el aborto remiten a múltiples cuestionamientos al poder y al orden social. Según Gutiérrez (2004), esto se debe a que el tema del aborto "pone en escena la problemática de la discusión moral y religiosa y su deslizamiento hacia lo jurídico (...) desnuda la problemática de explicar la escisión placer/reproducción (...) revierte la lógica de una sexualidad normativa y supuestamente natural” (p. 131).

El desconocimiento también puede estar relacionado a los "códigos culturales y estrechas restricciones y prohibiciones a la sexualidad femenina" impuestas por la influencia hispano-católica que ha sobrevivido por siglos en Puerto Rico y que sienta las pautas en el discurso y en el silencio sobre la sexualidad (Hernández-Angueira, 2008, p. 146; Pacheco, Rincón, Guevara, y Enriquez, 2007). De hecho, la mayoría de las personas que decían estar de acuerdo con la PCM admitían que su postura se fundamentaba en una moralidad religiosa. Además, tal como lo indica la literatura, el aborto a pesar de ser legal en Puerto Rico continúa estigmatizado (Colon-Warren, 2001) y acentuado por un alto grado de criminalización y clandestinidad. Por ejemplo, en Puerto Rico no se ofrecen servicios de aborto como un plan de salud pública por parte del Estado, ya que la mirada de la que se parte es política y religiosa, no salubrista. Esa visión salubrista estuvo casi ausente en nuestros hallazgos, lo que al parecer es general en Puerto Rico. En el portal Mujeres hoy $(2004,914)$, Azize expresa:

Yo creo que no sólo el tema del aborto, sino el de los anticonceptivos en general, carecen de una visión salubrista y de una visión apoyada en el derecho a la mujer a escoger, en apoyar a la mujer a prevenir embarazos no deseados y también en involucrar más al hombre para que asuma más responsabilidad.

Daros y colaboradores (2007, p.435) nos recuerdan que en "los debates al respecto [aborto] (...) las participaciones suelen adoptar posiciones de notoriedad, donde imperan más criterios políticos y de poder, muy lejos de lo que es estrictamente social". Es posible que el desconocimiento de la PCM limitara la opinión de los y las participantes sobre la misma. La mayoría de las personas enfocaron sus respuestas en las prácticas abortivas y no en la asignación de fondos para educación, consejería y prevención; contestando en base a sus juicios valorativos y morales. En nuestro estudio no indagamos directamente sobre si las personas están de acuerdo o no con el aborto ya que le tema central era la PCM; sin embargo, en otros estudios realizados en Puerto Rico encontraron que la mayoría estaban en contra del aborto, al menos a nivel discursivo (Chéverez-Rodríguez, 1987; Hernández-Angueira, 2008; Villarini-Baquero, 1994). Posiblemente, un conocimiento más profundo de la Política hubiese resultado en una mayor diversidad de opiniones; sin embargo, como sustenta Azize y Avilés (2009, J16): "la retórica de los líderes políticos y religiosos en contra del derecho a escoger ha dominado el debate público en torno al aborto". 
En términos de la relación entre algunas de las características socio-demográficas - edad, sexo, nivel de educación, ideología política, entre otros - y la actitud en torno a la planificación familiar, nuestros resultados sugieren que no existe relación entre estas. Estas variables fueron escogidas debido a que en estudios previos se sustenta su importancia en relación a la actitud hacia el aborto y las prácticas e ideologías sobre sexualidad (Azize y Avilés, 2009; Chéveres-Rodríguez, 1987; Hernández-Angueira, 2008; Saez, et al., 1993; Pacheco, et al., 2007; Villarini-Baquero, 1994). A excepción del trabajo de Villarini-Baquero (1994), en los demás estudios se identificó que estas variables, en especial edad y sexo, tienen un peso importante. Este dato no fue corroborado en nuestra investigación, sin embargo, mientras que en los estudios antes mencionados se realizaron análisis cualitativos y estadísticas descriptivas, en este análisis partimos de estadísticas inferenciales. Otro factor que pudiera estar vinculado a las diferencia en los resultados pudiera ser el tipo de muestra seleccionada, pues algunos fueron con estudiantes universitarios, otros con mujeres y en otros se limitaba el periodo de edad. Sin embargo, estudiosos y estudiosas del temas de género y sexualidad, entre los que se destaca Briggs (1964/2002), sustentan que estos están vinculados entre sí y, a su vez, están estrechamente ligados a la historia colonial de Puerto Rico y a la intervención sobre el cuerpo por parte del Estado, la Ciencia y la Iglesia.

La única variable socio-demográfica que tuvo una relación estadísticamente significativa con actitud fue la ideología religiosa, resultado congruente con el énfasis en la moral que las personas expresaron al posicionarse sobre el tema en la Fase I. Encontramos que tener una actitud altamente desfavorable hacia la planificación familiar estuvo mayormente representado por las personas con alguna afiliación religiosa, mientras que el otro extremo - altamente favorable - estuvo mayormente representado por personas que indicaron no tener ninguna afiliación religiosa. Destacamos que las personas que se identificaron como católicas mostraron una actitud más desfavorable hacia la PF que las personas protestantes. Esto guarda relación con la literatura revisada en la que se destaca que las explicaciones para sustentar y reincorporar la PCM es defendida con argumentos moralistas, dato que puede observarse en el acta misma de la PCM (CEPAL, 2000). Varas-Díaz (2010), en sus estudios sobre estigma y salud, ha identificado una relación entre ideología religiosa y los procesos de estigmatización a tal punto que plantea que la religión organizada es un problema de salud pública en Puerto Rico.

A partir de lo anterior, no es sorprendente que al evaluar las alternativas propuestas a la PF se inclinaran más hacia una perspectiva de prevención y que rechazaran el aborto como alternativa, lo que coincide con que la ideología religiosa-moral y el pensamiento tradicional fueran imperantes en el discurso de las personas participantes. Por otra parte, algunas personas ofrecieron posibles alternativas en torno a la dimensión económica del fenómeno bajo estudio. Esto concuerda con lo identificado en la literatura sobre Puerto Rico que sustenta que una de las razones para practicarse un aborto es precisamente problemas económicos, los que, a su vez, en muchas ocasiones impulsan a las mujeres a realizarse abortos en condiciones de riesgo por el alto costo de los mismos en las clínicas legales (Azize y Henshaw, 2003). Otra de las razones es la falta de conocimientos y del manejo adecuado de métodos anticonceptivos (Colón-Warren, 2001). De hecho, participantes en el estudio expresaron que hace falta más información y orientación por parte del gobierno y agencias relacionadas para prevenir los embarazos involuntarios a causa de la desinformación que, a fin de cuentas, terminan desembocando en abortos inducidos. Aun así, se entiende que existen múltiples factores adicionales a los evaluados a través de esta investigación que influyen en la actitud de una persona en torno a un tema de tal envergadura. Precisamente, una parte valiosa de estudio son las alternativas propuestas por algunas de las personas participantes para 
una política pública sobre planificación familiar que sea cónsona con la realidad social y cultural en Puerto Rico.

\section{Conclusiones}

En esta investigación exploramos los conocimientos, opiniones y actitudes sobre la planificación familiar y el aborto en Puerto Rico, a partir de la PCM. Este estudio fue una experiencia para desarrollar en estudiantes sub-graduados la motivación y conocimientos para realizar futuras investigaciones. Además, mediante este estudio pudimos identificar alternativas para desarrollar una política pública de PF desde las voces de las personas; por ejemplo, creación de planes integrales de orientación y de programas para personas de escasos recursos, talleres vivenciales y más educación en las escuelas. Al proveerles información sobre PF y la PCM a las personas participantes, aportamos a generar conocimiento relevante sobre estos temas en el contexto de la población puertorriqueña, lo cual puede ser útil para continuar desarrollando política pública al respecto.

Aunque esta investigación tiene varias limitaciones (i. e., muestra no probabilística ni representativa, tamaño de la muestra para fase cuantitativa, validación de instrumentos), indaga sobre un tema poco estudiado en Puerto Rico. Recomendamos validar los instrumentos creados para este estudio y replicarlo con una muestra probabilística, así como continuar explorando el tema. La política pública sobre el aborto en Puerto Rico sigue siendo un asunto inconcluso dentro de la agenda de la política pública de salud sexual y reproductiva (Azize y Avilés, 2009). Según Azize y Avilés $(2009$, I32):

La historia del aborto en Puerto Rico aún comparte realidades contradictorias que tienen su raíz en nuestra historia colonial. Estas contradicciones son el producto de casi seis décadas de políticas públicas erráticas - en los campos de la salud pública y poblacional - las cuales están estrechamente relacionadas con nuestra situación política.

\section{Agradecimientos}

Les expresamos el más profundo agradecimiento al grupo de profesionales ${ }^{3}$ que nos apoyaron en la realización de la investigación y al grupo de estudiantes ${ }^{4}$ de psicología que fueron parte integral en el éxito de la misma.

\footnotetext{
3 Elí Rodríguez-Cordero, Denisse Fonseca-Lago y Rafael Rivera-Ortiz.

4 Andrea Díaz, Ángel Alicea, Ángel Martínez, Ángeles Rodríguez, Angélica Caraballo, Bernice Morales, Carmen Morales, Chrystine Martínez, Delimar Santiago, Enid Otero, Faviana Silva, Irmaris Ríos, Jaime Colón, Joshua Sánchez, Jeylinne Enríquez, Jeanette Alejandro, Julyanna Torres, Keishalee Gómez, Karissa Hernández, Karla Vallejo, Laura Torres, Lizdalia Marcano, Lorna Sostre, María Díaz, María Lebrón, María Vélez, Mariana Coronado, Marilyn Rodríguez, Mayra Solis, Michelle Méndez, Mónica Rivas, Natalia Ramírez, Priscilla Delgado, Sandra Pabón, Sebastián León, Sohami Toledo, Sonia Rosado, Stephanie Cuebas, Vanesa Kelly, Verónica González, Verónica Muñiz, Victoria Rodríguez, Vivian Fontanés, Yesica Centeno y Yohalis Morales.
} 


\section{Referencias}

Acosta, Enrique; Carballo, Serafín; Corces, Valentín; Daza, M.a Teresa; Hernández, Felipe y Ubago, Carmen. (1982). Un enfoque interdisciplinario de planificación familiar. En Papeles del Psicólogo. Recuperado de http://www.papelesdelpsicologo.es/vernumero. asp?id=69

Asamblea Legislativa de Puerto Rico. (2004). Código Penal del Estado Libre Asociado de Puerto Rico, Ley Núm. 149. Recuperado de http://www.unifr.ch/ddp1/derechopenal/ legislacion/1_20080626_12.pdf

Azize, Yamila y Avilés, Luis A. (2009). Aborto en Puerto Rico: Límites de la Legalidad Colonial. Recuperado del Portal Saludpromujer de http://saludpromujer.org/index .php?option= com_content\&task=view\&id=45\&Itemid $=73$

Azize, Yamila y Henshay, Stanley (2003). Study of Induced Abortion in Puerto Rico, 20012002: Statistical and Demographic Information.Recuperadode http://saludpromujer.org/

Briggs, Laura (1964/2002). Race, Sex, Science and U.S. Imperialism in Puerto Rico: Reproducing Empire. Berkeley y Los Ángeles, California: University of California, Press.

Chéveres-Rodríguez, Nydia E. (1987). Conocimientos y Actitudes Hacia el Aborto por Demanda de un Grupo de Adolescentes Puertorriqueños (Tesis de Maestría sin publicar). Escuela Graduada de Salud Pública: Universidad de Puerto Rico- Río Piedras.

Clinton, William J. (1993). Memorandum for the acting administrator of the Agency for International Development. Recuperado de http://clinton6.nara.gov/1993/01/1993-0122-aid-family-planning-grants-mexico-city-policy.html

Colón-Warren, Alice E. (2001). Las políticas públicas sobre el aborto en países del Caribe Hispano. En panel Construcción y reconstrucción de identidades en la formación de políticas públicas en América Latina, el Caribe y U.S.A. Washington, D.C: Reunión de la Latin American Studies Association.

Comisión Económica para América Latina y el Caribe [CEPAL]. (2000). Listado de cumbres. Recuperado de http://www.eclac.cl/cumbres/listado_cumbres.asp

Creswell, John W. y Plano-Clark, Vicki L. (2007). Designing and Conducting Mixed Methods Research. California, EEUU: Sage Publications.

Daros, William R., Contreras Nieto, Miguel A., y Secchi, Mario A. (2007). La percepción social de los derechos del otro. Rosario, Argentina: Editorial CUI, UCEL, IUNIR.

Enciclopedia Británica. (2011). Welfare State. Recuperado de http://www.britannica.com/EB checked/topic/639266/welfare-state 
Indymediapr.org. (2010, 28 de septiembre). La Juntilla exige al gobierno que eduque a mujeres sobre salud sexual y reproductiva. Recuperado de http://pr.indymedia.org/news/2010/09/ 45164.php

Gutiérrez, María A. (2004). Silencios y susurros: La cuestión de la anticoncepción y el aborto. En Carlos F. Cáceres, Timothy Frasca, Mario Pecheny y Veriano Terto Júnior (Eds.). Ciudadanía sexual en América Latina: Abriendo el debate (pp. 129-139). Lima, Perú: Universidad Peruana Cayetano Heredia.

Hernández Angueira, Luisa (2008). El imaginario de la sexualidad y la representación de género en Puerto Rico, 1950-20001. Revista de Ciencias Sociales, 19, 128-149.

Mujeres Hoy: El portal de las mujeres latinoamericanas. (2004). Puerto Rico: entre la política pública y a legalidad del aborto. Recuperado de http://www.mujereshoy.com/ secciones/1878.shtm

Movimiento Amplio de Mujeres de Puerto Rico [MAMPR]. (2009). Movimiento amplio de mujeres no comparecerá a las vistas de confirmación de la Procuradora de las Mujeres. Recuperadodehttp://movimientoampliodemujeres.blogspot.com/2009_08_19_archive.html

NotiUno. (2009a, 30 de abril). Cuelgan procuradora de las mujeres: pregunta por Noti Uno fue principal detonante según senador popular. Recuperado de http://www.notiuno.com/ 2009/04/cuelgan-procuradora-de-las-mujeres-pregunta-de-noti-uno-fue-principaldetonante-segun-senador-popular/

NotiUno. (2009b, 21 de agosto). Confirman a Yvonne Feliciano como la nueva Procuradora de la Mujer. Recuperado de http://www.notiuno.com/2009/08/confirman-a-yvonnefeliciano-como-la-nueva-procuradora-de-la-mujer/

On The Issues. (2010). Ronald Reagan on Welfare \& Poverty. Recuperado de http://www.onthe issues.org/celeb/Ronald_Reagan_Welfare_+_Poverty.htm

Organización Mundial de la Salud. (2004). Global and Regional Estimates of the Incidence of Unsafe abortion and Associated Mortality in 2000 [4ta Ed.]. Recuperado de http:// whqlibdoc.who.int/publications/2004/9241591803.pdf

Pacheco, Carlos I., Rincón, Johanna, Guevara, Elías y Enríquez, Carolina. (2007) Socialización Sexual de Adolescentes Mujeres de 10 a 14 años: Un estudio de caso en la ciudad de Bogotá. Otras Miradas, 7(1), pp.29-29.

Population Reports (1999). Por qué es importante la planificación familiar. Recuperado de http:// info.k4health.org/pr/prs/sj49/j49chap1 .shtml

Smitha, Frank E. (2011). Postwar Welfare State to Thatcher and Reagan. En Macrohistory and World Report. Recuperado de http://www.fsmitha.com/h2/ch37-econ1a.htm 
Torres Degró, Arnaldo. (2004). La Políticas Poblacionales en Puerto Rico: Cinco siglos de dominación colonial. Universidad Complutense de Madrid. Recuperado de: http://www.ucm.es/BUCM/tesis/fsl/ucm-t27463.pdf

Varas-Díaz, Nelson (2010, noviembre). La religión organizada como un problema de salud pública.Primera Jornada de Psicología y Ateísmo:repensando debates centenarios para el siglo XXI, Universidad de Puerto Rico, Río Piedras, Puerto Rico.

Villarini-Baquero, Daniel. (1994). Actitudes y Conocimiento de una Muestra de Estudiantes del Recinto de Río Piedras de la Universidad de Puerto Rico acerca del Aborto (Tesina de Bachillerato sin Publicar). Facultad de Estudios Generales: Universidad de Puerto Rico-Río Piedras.

Wallace, Sarah K. (2008). The Domestic Roots of Reagan's Global Gag Rule: A Case Study in Global Health Diplomacy. Recuperado de http://www.thepresidency.org/storage/ documents/Fellows2008/Wallace.pdf 
